\title{
Tail Probability of Avoiding Poisson Traps for Branching Brownian Motion
}

\author{
Mehmet Öz \& Mine Çă̆lar \\ Department of Mathematics, Koç University, Istanbul, Turkey
}

\begin{abstract}
We consider a branching Brownian motion $Z$ with exponential branching times and general offspring distribution evolving in $\mathbb{R}^{d}$, where Poisson traps are present. A Poisson trap configuration with radius $a$ is defined to be the random subset $K$ of $\mathbb{R}^{d}$ given by $K=\bigcup_{x_{i} \in \operatorname{supp}(M)} \bar{B}\left(x_{i}, a\right)$, where $M$ is a Poisson random measure on $\mathbb{B}\left(\mathbb{R}^{d}\right)$ with constant trap intensity. Survival up to time $t$ is defined to be the event $\{T>t\}$ with $T=\inf \left\{s \geq 0: Z_{s}(K)>0\right\}$ being the first trapping time. Following the work of Engländer (2000, 2003), where strictly dyadic branching is considered, we consider here a general offspring distribution for $Z$ and settle the problem of survival asymptotics for the system.
\end{abstract}

Keywords: branching process, random environment, Poisson traps, tail probability

\section{Introduction}

We consider a branching Brownian motion evolving in $\mathbb{R}^{d}$ (Ikeda et al., 1968; Dawson, 1993; Engländer, 2000). We start with a single particle at the origin at $t=0$, performing Brownian motion for a random time which is distributed exponentially with constant parameter $\beta$. Then, the particle dies and simultaneously gives birth to a random number of particles distributed according to the offspring distribution $\mu$, which is a probability measure on $\mathbb{N}$. Similarly, each offspring particle repeats the same procedure independently from all others, starting from the position of her parent. In this way, one obtains a measure-valued Markov process $Z=\left(Z_{t}\right)_{t \geq 0}$, which is a particle configuration on $\mathbb{R}^{d}$. We assume that $Z_{0}=\delta_{0}$. The total mass process 
$|Z|=\left(\left|Z_{t}\right|\right)_{t \geq 0}$ is a continuous time $\mu$-Galton-Watson process with branching rate $\beta$. We denote the extinction time of the process $|Z|$ by $\tau$, which is formally defined as $\tau=\inf \left\{t \geq 0:\left|Z_{t}\right|=0\right\}$, where we use the convention that $\inf \emptyset=\infty$. We then denote the event of extinction of the process $|Z|$ by $E$, and define $E=\{\tau<\infty\}$. We use the term non-extinction for the event $E^{c}$, and save the term survival for the context of trapping. We let $P$ be the probability for the process $Z$.

The branching Brownian motion is assumed to live in a random environment consisting of Poisson traps. Let $M$ denote the Poisson random measure on $\mathbb{B}\left(\mathbb{R}^{d}\right)$ with mean measure $\nu=v \cdot$ Leb, where Leb stands for the Lebesgue measure, and $v>0$. A random trap configuration $K$ with radius $a$ on $\mathbb{R}^{d}$ is defined as

$$
K=\bigcup_{x_{i} \in \operatorname{supp}(\mathrm{M})} \bar{B}\left(x_{i}, a\right)
$$

where $\bar{B}\left(x_{i}, a\right)$ is the closed ball of radius $a$ centered at $x_{i}$. We let $\mathbb{P}$ be the probability for the Poisson traps, and $\mathbb{E}$ the corresponding expectation.

In this paper, we are interested in the probability that the branching system avoids the traps up to time $t$ asymptotically, averaged over all trap configurations. Let $T$ denote the first hitting time of $K$ by the branching Brownian motion $Z$ given by

$$
T=\inf \left\{s \geq 0: Z_{s}(K)>0\right\} .
$$

Survival up to time $t$ is defined by the event $\{T>t\}$. Our object of interest is the annealed (averaged) survival probability of the system up to time $t$ over all trap configurations, denoted by $\mathbb{E} \times P\{T>t\}$. We investigate its asymptotic behavior as $t \rightarrow \infty$ for various cases of the distribution $\mu$. The goal is to exhaust all possibilities for $\mu$.

\section{Previous results on survival asymptotics}

There are two major results for the survival asymptotics at hand, corresponding to two different deterministic instances of $\mu$.

Donsker and Varadhan (1975) studied the asymptotic behavior of the volume of the Wiener sausage. The Wiener sausage with radius $a$ is defined as

$$
W_{t}^{a}=\bigcup_{0 \leq s \leq t} \bar{B}(W(s), a)
$$


where $W$ stands for the Wiener process in $\mathbb{R}^{d}$. Its asymptotic distribution is given by

$$
\lim _{t \rightarrow \infty} t^{-\frac{d}{d+2}} \log E \exp \left(-v\left|W_{t}^{a}\right|\right)=-c(d, v),
$$

where $c(d, v)$ is a constant depending on $v>0$ and the dimension $d$. Letting $\left|W_{t}^{a}\right|$ be the volume of the Wiener sausage, by using the definition of Poisson random measure and applying Fubini's theorem, one observes that $E \exp \left(-v\left|W_{t}^{a}\right|\right)=\mathbb{E} \times P\{\bar{T}>t\}$ for $t>0$, where $\bar{T}$ is the analogue of $T$ for a single Brownian particle. In view of this equivalence, the work of Sznitman (1998, Chp.4) is an alternative proof of (1) in the context of Brownian survival among Poisson traps. Note that this solves the problem at hand for the case $\mu(1)=1$, and the scaling is subexponential.

Engländer $(2000,2003)$ considered the problem for the strictly dyadic branching case, corresponding to $\mu(2)=1$. The result is

$$
\lim _{t \rightarrow \infty} \frac{1}{t} \log \mathbb{E} \times P\{T>t\}=-\beta \quad \text { for } \quad d \geq 2
$$

and

$$
\lim _{t \rightarrow \infty} \frac{1}{t} \log \mathbb{E} \times P\{T>t\}=\left\{\begin{array}{ll}
-\beta \frac{v}{\bar{v}} & \text { if } v \leq \bar{v} \\
-\beta & \text { if } v>\bar{v}
\end{array} \text { for } d=1\right.
$$

where $\beta$ is as before the exponential lifespan parameter for the particles in the system, and $\bar{v}=\frac{1}{2} \sqrt{\frac{\beta}{2}}$ is the critical Poisson intensity. We see that the scaling is now exponential as a result of branching, which was not present in the problem of Donsker and Varadhan (1975). Also, for $d \geq 2$, we see the dominating effect of branching over spatial motion, since the result only depends on a branching parameter.

\section{Survival asymptotics for general offspring distribution}

In this section, we consider all the possibilities for the offspring distribution $\mu$. Observe that a continuous time $\mu$-Galton-Watson process (with $\mu(1) \neq 1$ ) with lifespan parameter $\beta$ is equal in law to the continuous time $\lambda$ Galton-Watson process with lifespan parameter $\beta(1-\mu(1))$, where $\lambda(1)=0$ and $\lambda(j)=\frac{\mu(j)}{1-\mu(1)}$ for $j \neq 1$. Therefore, we may and do assume henceforth that $\mu(1)=0$ for all continuous time $\mu$-Galton-Watson processes considered. 
Now we state our main result. We note that the first two parts of the theorem follow directly from existing results and trivial comparisons, while the third part is a non-trivial result that requires the application of Lyons' result (1992) on the set of individuals with infinite line of descent.

Theorem 1. Let $Z$ be a BBM with offspring distribution $\mu$ and branching rate $\beta$. It is assumed that $\mu(1)=0$ and $\beta>0$. Let $f$ be the generating function and $\tau$ be the extinction time for the underlying $\mu$-Galton-Watson process, that is $f(x)=\sum_{j=0}^{\infty} \mu(j) x^{j}$, and $\tau=\inf \left\{t \geq 0:\left|Z_{t}\right|=0\right\}$, where we adopt the convention that $\inf \emptyset=\infty$. Then, the survival asymptotics for the problem of BBM among Poisson traps with constant trap intensity $v$ is given as follows:

1. If $\mu(0)=0$, then

$$
\lim _{t \rightarrow \infty} \frac{1}{t} \log \mathbb{E} \times P\{T>t\}=-\beta \quad \text { for } \quad d \geq 2
$$

and

$$
\lim _{t \rightarrow \infty} \frac{1}{t} \log \mathbb{E} \times P\{T>t\}=-\beta \quad \text { for } \quad d=1, v>\bar{v}
$$

$-\beta \leq \liminf _{t \rightarrow \infty} \frac{1}{t} \log \mathbb{E} \times P\{T>t\} \leq \limsup _{t \rightarrow \infty} \frac{1}{t} \log \mathbb{E} \times P\{T>t\} \leq-\beta \frac{v}{\bar{v}}$

for $d=1, v \leq \bar{v}$, where $\bar{v}=\frac{1}{2} \sqrt{\frac{\beta}{2}}$ is the critical trap intensity for $d=1$.

2. If $\mu(0)>0$ and $\sum_{j=0}^{\infty} j \mu(j) \leq 1$, then

$$
\lim _{t \rightarrow \infty} \mathbb{E} \times P\{T>t\}=\mathbb{E} \times P(T>\tau)>0 .
$$

3. If $\mu(0)>0$ and $\sum_{j=0}^{\infty} j \mu(j)>1$, then

$$
\lim _{t \rightarrow \infty} \mathbb{E} \times P\{T>t\}=\mathbb{E} \times P(T>\tau \mid E) P(E)>0
$$

and

$$
\begin{aligned}
-\beta & \leq \liminf _{t \rightarrow \infty} \frac{1}{t} \log \mathbb{E} \times P\left(T>t \mid E^{c}\right) \\
& \leq \limsup _{t \rightarrow \infty} \frac{1}{t} \log \mathbb{E} \times P\left(T>t \mid E^{c}\right) \leq-\beta\left(1-f^{\prime}(q)\right)<0
\end{aligned}
$$




$$
\text { for } \begin{aligned}
d \geq 2 & \text { and } d=1, v>\bar{v} \\
-\beta & \leq \liminf _{t \rightarrow \infty} \frac{1}{t} \log \mathbb{E} \times P\left(T>t \mid E^{c}\right) \\
& \leq \limsup _{t \rightarrow \infty} \frac{1}{t} \log \mathbb{E} \times P\left(T>t \mid E^{c}\right) \leq-\beta \frac{v}{\bar{v}}\left(1-f^{\prime}(q)\right)<0
\end{aligned}
$$

for $d=1, v \leq \bar{v}$, where $E$ is the event of extinction and $q:=P(E)$ for the underlying $\mu$-Galton-Watson process.

Proof. We prove case by case.

1. Let $d \geq 2$. In Engländer (2000), $\mu(0)=0$ as well and $\mu(2)=1$. Comparison of the strictly dyadic case (2) with the general case of $\mu(0)=0$ yields

$$
\limsup _{t \rightarrow \infty} \frac{1}{t} \log \mathbb{E} \times P\{T>t\} \leq-\beta
$$

since introducing extra independent particles to the system decreases the survival probability for every $t>0$ and for every trap configuration. Note that one way for the system to survive is to suppress branching while the single Brownian particle avoids the traps. As the branching and motion mechanisms are independent from each other, it follows that for every $t>0$

$$
\mathbb{E} \times P\{T>t\} \geq e^{-\beta t} \mathbb{E} \times P\{\bar{T}>t\}
$$

where $e^{-\beta t}$ is the probability that the particle does not branch before time $t$, and $\bar{T}$ is the trapping time of a Brownian particle. Since $\mathbb{E} \times P\{\bar{T}>t\}$ decays subexponentially as $t \rightarrow \infty$ due to (1) and the discussion that follows, we find that

$$
\liminf _{t \rightarrow \infty} \frac{1}{t} \log \mathbb{E} \times P\{T>t\} \geq-\beta .
$$

Now, (4) and (5) together imply the result for $d \geq 2$. The result for $d=1$ follows from (3) in a similar fashion.

2. Firstly, we see that $\lim _{t \rightarrow \infty} \mathbb{E} \times P\{T>t\}$ exists by the BolzanoWeierstrass theorem since $\mathbb{E} \times P\{T>t\}$ is non-increasing in $t$ and bounded below by 0 . Now, let us bound this probability from below by a positive number. For instance consider the following event: a single Brownian particle starting at the origin remains in $B(0,1)$ until the time of first branching which occurs in the time interval $[0,1]$ and gives zero offspring, and there are no traps in $B(0,1+a)$. Since the mechanisms for branching, motion, and 
trap configuration are independent from each other, this event has positive probability, and the desired result follows.

We now prove the equality. If $\mu(0)>0$ and $\sum_{j=0}^{\infty} j \mu(j) \leq 1$, then the process is non-supercritical and the probability of extinction for the underlying $\mu$-Galton-Watson process is 1 , that is $P(E)=1$. The extinction time for the process is defined as $\tau=\inf \left\{t \geq 0:\left|Z_{t}\right|=0\right\}$. We note that in this case $\tau<\infty$ almost surely. Now we fix a trap configuration $\omega$ and let $P^{\omega}$ denote the conditional probability given this configuration. First we find $\lim _{t \rightarrow \infty} P^{\omega}\{T>t\}$, and for that end write for every $t>0$,

$$
P^{\omega}\{T>t\}=P^{\omega}\{T>t, \tau>t\}+P^{\omega}\{T>t \geq \tau\} .
$$

Note that the first term on the right in (6) is bounded by $P^{\omega}\{\tau>t\}$, and that $\lim _{t \rightarrow \infty} P^{\omega}\{\tau>t\}=0$ by monotone convergence since $\tau<\infty$ almost surely. We now concentrate on the second term, and try to relate it to the quantity $P^{\omega}\{T>\tau\}$. The following holds for every $t$,

$$
P^{\omega}\{T>\tau\}=P^{\omega}\{t \geq T>\tau\}+P^{\omega}\{T>t \geq \tau\}+P^{\omega}\{T>\tau>t\} .
$$

We see that the left hand side is a constant, free of $t$, so remains the same when we take the limit $t \rightarrow \infty$. The third term on the right is bounded above by $P^{\omega}\{\tau>t\}$, hence goes to zero in the limit $t \rightarrow \infty$ since $\lim _{t \rightarrow \infty} P^{\omega}\{\tau>t\}=0$. The first term on the right is $P^{\omega}\{t \geq T \cap T>\tau\}=$ $P^{\omega}\{t \geq T \mid T>\tau\} P^{\omega}\{T>\tau\}$, and we see that $P^{\omega}\{t \geq T \mid T>\tau\}$ tends to zero in the limit $t \rightarrow \infty$ since $T>\tau$ implies that $T=\infty$ almost surely. Since we have shown that the first and third terms on the right tend to zero as $t \rightarrow \infty$, we conclude that

$$
P^{\omega}\{T>\tau\}=\lim _{t \rightarrow \infty} P^{\omega}\{T>t \geq \tau\} .
$$

This result and (6) imply that $\lim _{t \rightarrow \infty} P^{\omega}\{T>t\}=P^{\omega}\{T>\tau\}$. Now, observing that $P^{\omega}\{T>t\}$ for each $t$ is a random variable on the probability space for traps, and applying bounded convergence theorem, we arrive at

$$
\lim _{t \rightarrow \infty} \mathbb{E} \times P\{T>t\}=\mathbb{E} \times P\{T>\tau\} .
$$

3. If $\mu(0)>0$ and $\sum_{j=0}^{\infty} j \mu(j)>1$, then the process is supercritical and we have $0<P(E)<1$. We write the annealed survival probability as

$$
\mathbb{E} \times P\{T>t\}=\mathbb{E} \times P(T>t \mid E) P(E)+\mathbb{E} \times P\left(T>t \mid E^{c}\right) P\left(E^{c}\right) .
$$


Since the second term tends to 0 in the limit $t \rightarrow \infty$, the first statement follows similar to case 2 above.

We now consider $\mathbb{E} \times P\left(T>t \mid E^{c}\right)$. Firstly, the lower bound for the annealed survival probability in (5) holds also for this case by identical arguments. Explicitly, we have

$$
\liminf _{t \rightarrow \infty} \frac{1}{t} \log \mathbb{E} \times P\left(T>t \mid E^{c}\right) \geq-\beta .
$$

Next, we find an upper bound. Due to conditioning on non-extinction, we appeal to the following result (Lyons, 1992, Proposition 4.10) on surviving Galton-Watson processes:

Lemma 1. Let $X=\left(X_{n}\right)_{n \geq 0}$ be a Galton-Watson process with probability generating function $f$, and let $X^{*}=\left(X_{n}^{*}\right)_{n \geq 0}$ be such that $X_{n}^{*}$ is the number of particles in $n^{\text {th }}$ generation that have an infinite line of descent. Suppose that $X$ is supercritical, and let $q$ be the probability of extinction for $X$. Then, the law of $X^{*}$ given non-extinction is the same as that of a Galton-Watson process with probability generating function $f^{*}(x)=[f(q+\bar{q} x)-q] / \bar{q}$ where $\bar{q}=1-q$ is the probability of non-extinction for $X$.

We apply the lemma above to $f(x)=\sum_{j=0}^{\infty} \mu(j) x^{j}$ with $\mu(0)>0$ and $\mu(1)=0$ to obtain

$$
\begin{aligned}
f^{*}(x)=\frac{1}{\bar{q}} & {\left[\mu(0)+\mu(2) q^{2}+\mu(3) q^{3}+\ldots-q\right.} \\
& \left.+\left(2 \mu(2) q \bar{q}+3 \mu(3) q^{2} \bar{q}+4 \mu(4) q^{3} \bar{q}+\ldots\right) x+\ldots\right] .
\end{aligned}
$$

Note that the constant term vanishes as $q$ solves the equation $x=\sum_{j=0}^{\infty} \mu(j) x^{j}$. Since the constant term vanishes, case 1 of the theorem is applicable. The coefficient of the linear term is the probability of giving 1 offspring for the process $Z^{*}$ and equals $f^{\prime}(q)$. As the tree generated by $Z^{*}$ is a subtree of that of $Z$, at any given time $t,\left|Z_{t}\right| \geq\left|Z_{t}^{*}\right|$. Then, by comparison with the process $Z^{*}$, and using the remark before the theorem on reduction to $\mu(1)=0$ case, we obtain an upper bound for the survival asymptotics of $Z$ for $d \geq 2$ :

$$
\limsup _{t \rightarrow \infty} \frac{1}{t} \log \mathbb{E} \times P\left(T>t \mid E^{c}\right) \leq-\beta\left(1-f^{\prime}(q)\right) .
$$

The result for $d=1$ follows from (3) by a similar argument. 
We check that $f^{\prime}(q)<1$. To see this, note that $f(x)=x$ at exactly one point in the interval $(0,1)$ and this point is $q$. This, together with the fact that $f(0)>0$ implies that $f(x)>x$ in the left-neighborhood of $q$. On the other hand, $f(x)<x$ in the right-neighborhood of $q$ since $f$ is increasing and convex on $[0,1]$ and $f(1)=1$. We conclude that $f^{\prime}(q)<1$.

Several remarks are in order. Case 1 in the theorem means that introducing more particles into the system by altering $\mu$ from $\mu(2)=1$ does not change the survival asymptotics for $d \geq 2$. The decay remains exponential asymptotically with the same rate $\beta$. Case 3 of the theorem implies that for supercritical $\mu$ with $\mu(0)>0$, the decay of the survival probability given non-extinction remains exponential asymptotically. We note that a special case of interest is when $\mu(0)>0, \mu(0)+\mu(2)=1$ and $\mu(2)>\mu(0)$. In this case since $f(x)=\mu(0)+\mu(2) x^{2}$ and $q=\frac{\mu(0)}{\mu(2)}, f^{\prime}(q)$ becomes $2 \mu(0)$ and we have

$$
\limsup _{t \rightarrow \infty} \frac{1}{t} \log \mathbb{E} \times P\left(T>t \mid E^{c}\right) \leq-\beta(\mu(2)-\mu(0))
$$

for $d \geq 2$. Finally, we conjecture that the relevant limits exist in Theorem 1 and leave as future work.

Acknowledgements. The authors would like to thank the anonymous reviewer for very constructive comments which improved the manuscript.

\section{References}

[1] M. D. Donsker, S. R. S. Varadhan: Asymptotics for the Wiener sausage, Communications in Pure and Applied Mathematics, 28 (4), 525 - 565, 1975 .

[2] J. Engländer: On the volume of the supercritical super-Brownian sausage conditioned on survival, Stochastic Processes and their Applications, 88, $225-243,2000$.

[3] J. Engländer and F. den Hollander: Survival asymptotics for branching Brownian motion in a Poissonian trap field, Markov Processes and Related Fields, 9 (3), 363 - 389, 2003.

[4] A. Sznitman: Brownian motion, obstacles and random media, Springer Monographs in Mathematics, Springer-Verlag, Berlin, 1998. 
[5] R. Lyons: Random walks, capacity and percolation on trees, Annals of Probability, 20 (4), 2043 - 2088, 1992.

[6] D.A. Dawson: Measure-valued Markov processes, École d'éte de probabilités de Saint Flour XXI, 1541, 1 - 260, 1993.

[7] N. Ikeda, M. Nagasawa and S. Watanabe: Branching Markov processes I,II,III, J. Math. Kyoto Univ., 8, 233-278, 365-410; 9, 95-160, 1968,1969. 